CIC. Cuadernos de Información y Comunicación

ISSN: $1135-7791$

http://dx.doi.org/10.5209/CIYC.60913

\title{
La política da que hablar: Engagement en redes sociales de sitios de noticias
}

\author{
Eugenia Mitchelstein ${ }^{1}$; Silvana Leiva1; Camilia Giuliano'; Pablo J. Boczkowski²
}

Recibido: 9 de julio de 2018 / Aceptado: 14 de julio de 2018

Resumen. Este trabajo examina la relación entre los contenidos presentados en las cuentas oficiales de Facebook y Twitter de ocho sitios de noticias de Argentina y el nivel de engagement que generaron en los usuarios. El análisis de una muestra de 2.039 noticias, publicadas entre los meses de marzo a noviembre de 2017, muestra que existe una relación entre el contenido de las noticias y el nivel de engagement que las mismas generan entre los usuarios. Si bien los sitios presentaron una mayor cantidad de noticias sobre temáticas de asuntos no públicos, como deportes, policiales o entretenimiento, los mayores niveles de engagement se registraron en noticias sobre asuntos públicos, como política nacional e internacional y economía. Estos hallazgos son consistentes con la literatura sobre proceso de adaptación de los medios al nuevo ecosistema mediático y apoyan la teoría de "ciudadanía monitorial" en el contexto digital.

Palabras clave: engagement, redes sociales, sitios de noticias, asuntos públicos, preferencias de los lectores

\section{[en] The policy gives to speak: Engagement in social networks of news sites}

\begin{abstract}
This paper analyses the relationship between the contents presented in the official Facebook and Twitter accounts of eight news sites in Argentina and the level of engagement they generate among users. The analysis of a sample of 2,039 news items, published between the months of March to November 2017, shows that there is a correlation between the content of the news and the level of engagement that they create among users. Although the sites presented a greater amount of news on issues of non-public issues, such as sports, police or entertainment, the highest levels of engagement were recorded in news on public issues, such as national and international policy and economy. These findings are consistent with the literature on the process of adaptation of the media to the new media ecosystem and support the theory of "monitorial citizenship" in the digital context.
\end{abstract}

Keywords: engagement, social networks, news sites, public issues, readers preferences.

Sumario. La política da que hablar: Engagement en medios y redes sociales. Redes sociales: Consecuencias informativas y políticas. Método. Resultados. Análisis cuantitativo. Análisis cualitativo. Conclusiones. Referencias.

Cómo citar: Mitchelstein, E.; Leiva, S.; Giuliano, C.; Boczkowski, P. J. (2018). La política da que hablar: Engagement en redes sociales de sitios de noticias, en CIC. Cuadernos de Información y Comunicación 23, 157-173.

Universidad de San Andrés, Argentina.

Northwestern University, EE.UU. 


\section{La política da que hablar: Engagement en medios y redes sociales}

Los medios informativos se encuentran ante un proceso de migración y adaptación a nuevas formas de producción, distribución y circulación de noticias a raíz de la creciente popularidad de nuevas plataformas digitales, entre las que se cuentan las redes sociales. En la Argentina, 72\% de los consumidores accede a noticias a través de redes sociales, incluyendo $60 \%$ que lo hace a través de Facebook y $18 \%$ que accede a información periodística a través de Twitter (Newman et al., 2018). En España, estas cifras son del $60 \%, 48 \%$ y $22 \%$, respectivamente (Newman et al., 2018).

La literatura especializada ha analizado al menos dos tipos de implicaciones del consumo de noticias en redes sociales. Primero, hay estudios que se enfocan en las consecuencias para el periodismo y la difusión de información, y señalan que existe una correlación positiva entre el uso de redes y el acceso a noticias, promovida en parte por un aumento del consumo incidental de estas últimas en las redes (Boczkowski, Mitchelstein y Matassi, 2018; Fletcher y Nielsen, 2018; Mitchelstein y Boczkowski, 2018; Oeldorf-Hirsch y Sundar, 2015). Algunos autores relacionan el creciente acceso a la información a través de redes sociales con la pérdida de poder de fijación de agenda por parte de medios tradicionales y con cambios en la manera de presentar y distribuir la información (Conway-Silva, Filer, Kenski, y Tsetsi, 2017; Vargo, et al., 2018; Meraz, 2009). Segundo, otros especialistas se han concentrado en la relación entre el acceso a redes y el comportamiento político y social de los usuarios (De Zuñiga, Jung y Valenzuela, 2012; Oh y Syn, 2015). Por ejemplo, hay trabajos que encuentran un aumento en la participación política en función de las interacciones de los usuarios en redes sociales (Ardèvol-Abreu y Gil de Zúñiga., 2017; Valeriani y Vaccari, 2016).

Este texto se propone avanzar en el análisis sobre interacción entre medios de noticias y sus audiencias en redes sociales relacionando, por un lado, con los hallazgos previos en la materia que sugieren relaciones positivas entre uso de redes, el consumo informativo y participación política; y por el otro con los trabajos que identifican diferentes usos que los medios informativos les otorgan a sus redes sociales. Para ello examinamos la existencia de una posible correlación entre temática de las noticias y nivel de engagement generado por los usuarios en las cuentas oficiales de Facebook y Twitter de ocho sitios de noticias de la Argentina. El análisis cuantitativo se complementa con un estudio cualitativo exploratorio sobre qué tipo de contenido genera el mayor nivel de engagement. Los hallazgos muestran que, mientras que en sus cuentas de redes sociales los medios proponen una agenda más asociada a contenidos de asuntos no públicos, como deportes, policiales o entretenimiento, las noticias que más engagement generan en los usuarios son sobre asuntos públicos, como política nacional e internacional y economía. A partir de estos resultados, discutimos las implicancias para la distribución de contenido noticioso a través de redes sociales y su impacto en la esfera pública.

\section{Redes sociales: Consecuencias informativas y políticas}

En un ecosistema mediático convergente (Jenkins, 2008), las redes sociales exacerban el cambio en las formas en las que se produce y consume contenido online, ya que generan una abundancia de contenido sin precedentes (Asur, Huberman, Szabo, 
\& Wang, 2011), y nuevas formas de interacción con los medios, más participativas que las existentes en los medios tradicionales (Effing, Van Hillegersberg, \& Huibers, 2011). Las investigaciones han hecho hincapié en al menos dos consecuencias principales de la difusión de noticias en redes sociales.

En primer lugar, han estudiado las consecuencias periodísticas tanto en la creación de contenido como en la fijación de agenda. Los estudios señalan que los medios confían cada vez más en las redes sociales para volcar sus contenidos y llegar a sus audiencias (Powers y Vera-Zambrano, 2017; Wanta, Golan y Lee, 2004). Esto trae como consecuencia la co-creación de noticias entre periodistas y usuarios, y la personalización del contenido que los usuarios reciben a través de las redes (Stassen, 2010; Sunstein, 2018; Wasike, 2013). La mayoría de los trabajos sobre inserción periodística en redes señalan una tendencia al diálogo y a la interacción (Conway Kenski, y Wang, 2012).

Otros autores han analizado si existe una pérdida del poder de fijación de agenda de los medios tradicionales a partir de la difusión masiva de las redes sociales (Conway-Silva, et al., 2017; Guo y Vargo, 2018; Meraz, 2009). Los estudios indican que conforme ha aumentado el acceso a noticias a través de redes sociales (Gil de Zúñiga, Jung, y Valenzuela, 2012; Messing y Westwood, 2014), tanto deliberado como incidental (Boczkowski, Mitchelstein, y Matassi, 2018; Hermida, Fletcher, Korell y Logan, 2012), también se ha incrementado la influencia de las recomendaciones de amigos y otros contactos en redes sociales por encima de la lógica editorial propuesta por los medios tradicionales, tanto en sus sitios como en sus cuentas en redes (Turcotte, York, Irving, Scholl, y Pingree, 2015; Wasike, 2013; Weeks y Holbert, 2013).

En segundo lugar, la literatura especializada también ha examinado las implicaciones políticas del acceso a información en redes sociales, como el potencial de las redes para la movilización (Lim, 2012; Sakaki, Okazaki y Matsuo, 2010), y su impacto en los niveles de participación política de los usuarios (Effing, Van Hillegersberg, y Huibers, 2011). Valenzuela (2013) propone tres posibles usos "políticos" de las redes sociales: información, expresión de opiniones y activismo. Cada vez más, las redes sociales se convierten en un lugar en el que los usuarios expresan sus opiniones políticas (Rainie, Smith, Schlozman, Brady, y Verba, 2012). En Estados Unidos, $68 \%$ de los usuarios utiliza las redes sociales para consultar noticias, y un $66 \%$ para realizar actividades de participación política (Gottfried y Shearer, 2016).

Compartir y comentar noticias son rasgos que, si bien definen la interactividad de las redes sociales, ya se habían examinado en los sitios de medios de noticias (Boczkowski y Mitchelstein, 2012). Algunos especialistas sugieren que la función de compartir información puede percibirse como un tipo de comportamiento participativo que depende no solo de intereses particulares de los usuarios, sino también de otras motivaciones altruistas, sociales o individuales (Bachmann y Gil de Zúñiga, 2013; Gil de Zúñiga, Molyneux y Zheng, 2014; Nielsen y Schrøder, 2014). Se comparte información para ganar reputación, conseguir aprobación social y ganar "autoridad" (boyd, Golder, y Lotan, 2010; Lee y Ma, 2012; Ma, Lee, y Goh, 2011), pero también por razones más individuales como intercambiar información, entretenerse o contribuir a beneficios sociales (Holton, Baek, Coddington, y Yaschur, 2014; Martínez 2010). Estas motivaciones pueden variar, además, según las características de las redes sociales. Oh y Syn (2015) señalan que los usuarios de Facebook se encuentran mayormente motivados por el disfrute, el altruismo, el compromiso social y la 
reciprocidad, mientras que en Twitter predomina la reputación y la eficacia. Según los autores, la razón reside en el formato de cada plataforma, pues Twitter, como microblog, permite la autopromoción, mientras que Facebook permite actividades más orientadas a la relación con otros.

En suma, las motivaciones en el uso de redes sociales se asocian a las funciones que las redes permiten tanto como a las decisiones individuales (Quan-Haase y Young, 2010; Whiting y Williams, 2013). En este contexto, el concepto del engagement, como una medida que cuantifica el nivel de determinadas formas de "interacción” en redes sociales (Hollebeek et al., 2014; Raimondo Anselmino, 2017), aparece como relevante en el análisis sobre el consumo y la circulación de las noticias. Las investigaciones han mostrado que compartir noticias en redes tiene algún efecto deseable en términos de engagement: que se incrementa cuando el contenido es compartido públicamente y cuando el usuario consulta la opinión de otros además de compartir contenido (Oeldorf-Hirsch y Sundar, 2012). En estudios más centrados en la circulación de contenido en redes, el concepto de engagement se asocia a la "popularidad" expresada en el tiempo de vida de un contenido dentro de las redes o la cantidad de usuarios que la comparten. En este sentido, existen estudios que intentan encontrar atributos o patrones que hacen que un contenido se considere "popular" (Bandari et al., 2012) y han encontrado una correlación entre la popularidad alcanzada de contenidos de redes y su popularidad previa fuera de ellas (Lee, Moon, y Salamatian 2010).

Este trabajo se posiciona en el nodo que une los estudios sobre construcción de noticias y fijación de agenda y compromiso cívico en redes sociales para examinar qué temáticas obtienen mayores niveles de engagement en las cuentas oficiales de redes sociales de ocho sitios de noticias de Argentina. Se plantea dos preguntas de investigación principales:

P.I.1: ¿Cómo está compuesta la agenda de las cuentas oficiales de redes sociales de ocho sitios de noticias de la Argentina?

P.I.2: ¿Qué factores están relacionados con mayores niveles de engagement con las noticias en redes sociales?

\section{Método}

Este trabajo hace un análisis cuantitativo y cualitativo de una muestra de las noticias distribuidas por ocho sitios argentinos durante el período de marzo a noviembre de 2017 en sus cuentas oficiales de Facebook y Twitter. El corpus alcanzó un total de 2.039 noticias de los siguientes sitios: Clarín.com, Lanacion.com.ar, Perfil.com, Infobae.com, TN.com.ar, Lavoz.com.ar, Diariouno.com.ar y Pagina12.com.ar (Cuadro 1). Estos fueron elegidos por su relevancia y tradición en el sistema de medios argentino; todos ellos poseen un alcance nacional y un rol predominante en la fijación de agenda. Además, poseen una agenda temática generalista, amplia y heterogénea. Esta elección tiene importancia metodológica para nuestro trabajo en la medida que permite analizar comparativamente las temáticas que suscitan mayor popularidad entre los usuarios.

Clarín es la contraparte online del periódico con mayor circulación en la nación, con un promedio diario de 206.000 ejemplares, tiene un ranking Alexa de 13 en el país, y sus cuentas de Facebook y Twitter poseen 5.703.704 y 2.700.000 seguidores, 
Cuadro 1. Composición del corpus según cantidad de noticias por sitio y por red social.

\begin{tabular}{|l|c|c|c|}
\hline \multicolumn{1}{|c|}{ Sitio } & $\begin{array}{c}\text { Cantidad noticias } \\
\text { en Twitter }\end{array}$ & $\begin{array}{c}\text { Cantidad noticias } \\
\text { en Facebook }\end{array}$ & $\begin{array}{c}\text { Cantidad noticias } \\
\text { totales }\end{array}$ \\
\hline clarin.com & 139 & 130 & 269 \\
\hline lanacion.com.ar & 136 & 133 & 269 \\
\hline perfil.com & 137 & 130 & 267 \\
\hline infobae.com & 137 & 129 & 266 \\
\hline tn.com.ar & 132 & 128 & 260 \\
\hline lavoz.com.ar & 136 & 129 & 265 \\
\hline diariouno.com.ar & 137 & 129 & 177 \\
\hline pagina12.com.ar & 138 & 39 & 2.039 \\
\hline Total & 1.092 & 947 & \\
\hline
\end{tabular}

respectivamente. La Nación es la contraparte online del periódico con el segundo nivel de circulación más alto (117.000), tiene un ranking Alexa de 15 en Argentina, y sus cuentas de Facebook y Twitter tienen 3.537.826 y 3.120.000 seguidores, respectivamente. Perfil, también con base en Buenos Aires, es la versión online de un periódico en papel quincenal, con una circulación promedio de 25.780 ejemplares, tiene un ranking Alexa de 55 en Argentina, y sus cuentas de Facebook y Twitter tienen 623.829 y 738.000 seguidores, respectivamente. Página/12 es la contraparte online de un periódico impreso de orientación ideológica de izquierda con un promedio diario de circulación de 13.200 ejemplares, tiene un ranking Alexa de 50 en Argentina, y 473.660 y 117.000 seguidores respectivamente en sus cuentas de Facebook y Twitter. La Voz del Interior es la contraparte online del diario editado fuera de Buenos Aires más vendido, con base en la ciudad de Córdoba, cuya circulación promedio es de 37.000, posee un ranking Alexa 83 en Argentina, y sus cuentas de Facebook and Twitter poseen 1.024 .689 y 491.000 seguidores respectivamente. Diario Uno, la versión online de un diario publicado en la ciudad de Mendoza, es parte de un conglomerado multimedios, y posee una circulación promedio de 7.600. Este sitio posee un ranking Alexa de 284 en Argentina, y sus cuentas de Facebook y Twitter poseen 1.560.124 y 326.000 seguidores, respectivamente. Infobae es el principal sitio de noticias online de Argentina, tiene un ranking Alexa de 11, y sus cuentas de Facebook y Twitter poseen 2.477.308 y 997.000 seguidores, respectivamente. TN es la versión online de un canal de televisión de cable homónimo, la cual es la señal de cable con mayor audiencia y parte del mismo conglomerado mediático al cual pertenece el diario Clarín. Este sitio posee un ranking Alexa de 58 en Argentina, y sus cuentas de Facebook and Twitter poseen 6.916.515 and 4.640.000 seguidores, respectivamente. Todos estos datos son al mes de marzo de 2018 (IVC, 2018).

Para el armado del corpus tomamos una muestra de 14 días ensamblados según la metodología de la semana construida (Hester y Dougall, 2007; Krippendorff y Bock, 2009; Riffe, Aust y Lacy, 1993;), durante los meses de marzo y noviembre de 
$2017^{3}$. En cada uno de estos días se capturó un total de 10 noticias publicadas en las cuentas oficiales de Facebook y Twitter de los ocho sitios (10 por cada red social por día por sitio). La recolección de las noticias en cada uno de los días fue realizado mediante un software que agrega automáticamente las últimas 10 publicaciones de cada sitio en ambas redes a las 18:00 horas de cada uno de los días. Este horario fue seleccionado ya que, como señala la literatura al respecto, suele ser uno de los más apropiados para entender el flujo de información en redes sociales (Raimondo Anselmino, 2017). El análisis se realizó sobre el contenido de las noticias tal como aparecen en su sitio, a partir del link que figura en las redes sociales.

Las noticias fueron codificadas según cuatro variables principales:

1) Sitio de origen de cada noticia: Clarín, La Nación, Perfil, Infobae, TN, La Voz del Interior, Diario Uno, Página/12.

2) Red en la que se publicó cada noticia:

Facebook

Twitter

3) Tema:

Asuntos públicos: artículos sobre política, economía, figuras públicas con implicancia política explicita, tanto a nivel nacional como internacional.

Asuntos no públicos: aquellas noticias que no poseen vinculaciones explícitas con cuestiones políticas.

4) Nivel de engagement

Facebook: La suma de la cantidad de interacciones (Likes, Angry, Haha, Sad, Loves, Wow, Shares, Comments) registradas por posteo Facebook.

Twitter: la suma de la cantidad de interacciones (Likes, Retweets y Replies) registradas por posteo en Twitter., ${ }^{4,5}$

\section{Resultados}

\section{Análisis Cuantitativo}

En la muestra recogida para este estudio, los sitios presentaron, en promedio, tanto en Twitter como en Facebook, un mayor porcentaje de noticias sobre asuntos no públicos, aunque en Facebook se registra una mayor diferencia entre noticias de asuntos públicos y noticias de asuntos no públicos: en promedio, 69\% de las noticias publicadas en las cuentas de Facebook de los ocho sitios son de asuntos no públicos, comparadas con $62 \%$ de los artículos distribuidos en las cuentas de Twitter de los mismos ocho medios (Cuadro 2). En siete de los ocho sitios se verifica esta tenden-

3 Los catorce días de la muestra se construyeron a partir de la metodología de semana construida durante los meses de marzo, abril, mayo, junio, julio, agosto y noviembre. No representan meses corridos por la falta de datos de septiembre y octubre debido a un error en el sistema automatizado de recolección de información para la red social Facebook.

4 El intercoder reliability (Krippendorff \& Bock, 2009) de la variable "tema" fue del 94\%, con un índice Cohen Kappa de 0.88. Los niveles de engagement fueron registrados por el bot que recolectó las noticias y no por codificadores humanos.

5 Como dimensión de análisis, tomamos el concepto propuesto por Raimondo Anselmino (2017): "[la medición de engagement] se centra, directamente, en las cantidades y en los porcentajes de interacciones registradas". 
Cuadro 2. Porcentaje de asuntos públicos y asuntos no públicos en cada uno de los sitios, en ambas redes sociales.

\begin{tabular}{|c|c|c|c|c|}
\hline \multirow[b]{2}{*}{ Sitio } & \multicolumn{2}{|c|}{ Facebook } & \multicolumn{2}{|c|}{ Twitter } \\
\hline & $\begin{array}{l}\text { Asuntos } \\
\text { Públicos }\end{array}$ & $\begin{array}{c}\text { Asuntos } \\
\text { No Públicos }\end{array}$ & $\begin{array}{l}\text { Asuntos } \\
\text { Públicos }\end{array}$ & $\begin{array}{c}\text { Asuntos } \\
\text { No Públicos }\end{array}$ \\
\hline \multirow[t]{3}{*}{ Clarín } & $32 \%$ & $68 \%$ & $40 \%$ & $60 \%$ \\
\hline & $\mathrm{N}=41$ & $\mathrm{~N}=89$ & $\mathrm{~N}=55$ & $\mathrm{~N}=84$ \\
\hline & \multicolumn{2}{|c|}{$t(129)=-9,0247, p=0,000$} & \multicolumn{2}{|c|}{$t(138)=-5,0117, p=0,000$} \\
\hline \multirow[t]{3}{*}{ La Nación } & $44 \%$ & $56 \%$ & $30 \%$ & $70 \%$ \\
\hline & $\mathrm{N}=56$ & $\mathrm{~N}=77$ & $\mathrm{~N}=41$ & $\mathrm{~N}=95$ \\
\hline & \multicolumn{2}{|c|}{$t(132)=-3,6731, p=0,0003$} & \multicolumn{2}{|c|}{$t(135)=-10,0525, p=0,000$} \\
\hline \multirow[t]{3}{*}{ Perfil } & $35 \%$ & $65 \%$ & $40 \%$ & $60 \%$ \\
\hline & $\mathrm{N}=46$ & $\mathrm{~N}=84$ & $\mathrm{~N}=55$ & $\mathrm{~N}=82$ \\
\hline & \multicolumn{2}{|c|}{$t(129)=-6,3623, p=0,000$} & \multicolumn{2}{|c|}{$t(136)=-4,6877, p=0,000$} \\
\hline \multirow[t]{3}{*}{ Infobae } & $37 \%$ & $63 \%$ & $36 \%$ & $64 \%$ \\
\hline & $\mathrm{N}=48$ & $\mathrm{~N}=81$ & $N=49$ & $\mathrm{~N}=88$ \\
\hline & \multicolumn{2}{|c|}{$t(128)=-5,9875, p=0,0000$} & \multicolumn{2}{|c|}{$t(136)=-6,9253, p=0,000$} \\
\hline \multirow[t]{3}{*}{$T N$} & $27 \%$ & $73 \%$ & $32 \%$ & $68 \%$ \\
\hline & $\mathrm{N}=34$ & $\mathrm{~N}=94$ & $\mathrm{~N}=42$ & $\mathrm{~N}=90$ \\
\hline & \multicolumn{2}{|c|}{$t(127)=-11,9611, p=0,0000$} & \multicolumn{2}{|c|}{$t(131)=-8,9353, p=0,0000$} \\
\hline \multirow[t]{3}{*}{ La Voz } & $23 \%$ & $77 \%$ & $34 \%$ & $65 \%$ \\
\hline & $\mathrm{N}=30$ & $\mathrm{~N}=99$ & $\mathrm{~N}=48$ & $\mathrm{~N}=88$ \\
\hline & \multicolumn{2}{|c|}{$t(128)=-14,3233, p=0,0000$} & \multicolumn{2}{|c|}{$t(135)=-7,1520, p=0,0000$} \\
\hline \multirow[t]{3}{*}{ Diario Uno } & $11 \%$ & $89 \%$ & $18 \%$ & $82 \%$ \\
\hline & $\mathrm{N}=14$ & $\mathrm{~N}=115$ & $\mathrm{~N}=25$ & $\mathrm{~N}=112$ \\
\hline & \multicolumn{2}{|c|}{$t(128)=-28,4792, p=0,0000$} & \multicolumn{2}{|c|}{$t(136)=-19,1733, p=0,000$} \\
\hline \multirow[t]{3}{*}{ Página/12 } & $74 \%$ & $26 \%$ & $76 \%$ & $24 \%$ \\
\hline & $\mathrm{N}=29$ & $\mathrm{~N}=10$ & $\mathrm{~N}=105$ & $\mathrm{~N}=33$ \\
\hline & \multicolumn{2}{|c|}{$t(38)=6,8779, p=0,000$} & \multicolumn{2}{|c|}{$t(137)=14,3175, p=0,000$} \\
\hline \multirow[t]{3}{*}{ Total } & $31 \%$ & $69 \%$ & $38 \%$ & $62 \%$ \\
\hline & $\mathrm{N}=298$ & $\mathrm{~N}=649$ & $\mathrm{~N}=420$ & $\mathrm{~N}=672$ \\
\hline & \multicolumn{2}{|c|}{$t(946)=-24,7191, p=0,0000$} & \multicolumn{2}{|c|}{$t(1091)=-15,6687, p=0,0000$} \\
\hline
\end{tabular}


cia. En Facebook, los porcentajes van de 56\% de posteos sobre asuntos no públicos en la cuenta oficial de La Nación, a 89\% en la cuenta de Diario Uno. En Twitter, 6 de cada diez de las noticias posteadas por Perfil y Clarín son sobre asuntos no públicos, comparadas con nueve de cada diez de las posteadas por Diario Uno. Página/12 aparece como una excepción en las dos redes sociales examinadas, mostrando una mayoría de noticias sobre asuntos públicos tanto en Facebook como en Twitter (26\% y 24\%, respectivamente). En los casos de Clarín, Perfil, TN, La Voz del Interior y Diario Uno, Facebook es la red en donde se publicó la mayor cantidad de noticias sobre temas de asuntos no públicos, en tanto La Nación es el único sitio que publicó más noticias sobre asuntos públicos en Facebook que en Twitter, mientras que Infobae mostró porcentajes muy similares en las dos redes sociales (63\% y 64\%), respectivamente. En respuesta a la P.I.1, los medios de noticias en redes sociales presentan una agenda en la que predominan los asuntos no públicos.

Para dar cuenta del nivel de interacción entre los usuarios y el contenido al que son expuestos, analizamos los niveles de engagement conseguidos por las noticias en ambas redes sociales (Cuadro 3). El promedio de engagement en Facebook es de 241 , siendo el valor máximo 12.958 y el valor mínimo 0 . En tanto, en Twitter el promedio de engagement es de 32, siendo el valor máximo 708 y el mínimo 0. Estos resultados están en consonancia con la relativa difusión de ambas redes sociales en la Argentina y su popularidad como fuentes de investigación periodística. En Facebook el tipo de interacciones más frecuente fueron las "reacciones", mientras que en Twitter parece haber una distribución más pareja entre los tres tipos de interacción que ofrece la plataforma. En las cuentas de estos ocho sitios, Facebook genera siete veces más instancias de engagement, en promedio, que Twitter.

Para responder a la P.I.2, se realizaron regresiones lineales con "nivel de engagement" como variable dependiente, y tema y sitio como variables independientes (Cuadro 4). La relación entre el contenido de asuntos públicos y el nivel de engagement de las noticias tanto en Facebook como en Twitter es positiva, controlando por sitio. Específicamente, en el caso de Facebook, esto implica que un aumento de una

Cuadro 3. Engagement de noticias en Facebook y Twitter desagregado por tipo de interacción (todos los sitios incluidos).

\begin{tabular}{|l|r|r|c|c|}
\cline { 2 - 5 } \multicolumn{1}{c|}{} & Media & Mínimo & Máximo & $\begin{array}{c}\text { Desviación } \\
\text { Estándar }\end{array}$ \\
\hline Total observaciones Facebook $(\mathbf{N}=\mathbf{9 5 3})$ & 241 & 0 & 12.958 & 755,30 \\
\hline Reacciones Facebook & 94 & 0 & 10.788 & 423,81 \\
\hline Shares Facebook & 31 & 0 & 3.077 & 201,74 \\
\hline Comentarios Facebook & 7 & 0 & 602 & 37,31 \\
\hline Total observaciones Twitter $(\mathbf{N}=\mathbf{1 0 9 6})$ & 32 & 0 & 708 & 53,75 \\
\hline Likes Twitter & 4 & 0 & 206 & 12,47 \\
\hline RTs Twitter & 11 & 0 & 164 & 19,70 \\
\hline Replies Twitter & 17 & 0 & 462 & 30,77 \\
\hline
\end{tabular}


Cuadro 4. Regresiones lineales de "Engagement Twitter" y "Engagement Facebook" sobre asuntos públicos y sitio (caso base: Clarín)

\begin{tabular}{|c|c|c|}
\hline Variables & Engagement Facebook & Engagement Twitter \\
\hline \multirow[t]{2}{*}{ Asuntos públicos } & $174,2 * * *$ & $12,00 * * *$ \\
\hline & $(53,26)$ & $(1,977)$ \\
\hline \multirow[t]{2}{*}{ La Nación } & 83,50 & $6,602 *$ \\
\hline & $(89,95)$ & $(3,505)$ \\
\hline \multirow[t]{2}{*}{ Perfil } & $-402,7 * * *$ & $-3,300$ \\
\hline & $(90,67)$ & $(3,512)$ \\
\hline \multirow[t]{2}{*}{ Infobae } & $-387,5^{* * *}$ & -0.522 \\
\hline & $(90,51)$ & $(3,515)$ \\
\hline \multirow[t]{2}{*}{$T N$} & $-187,5^{* *}$ & $17,18 * * *$ \\
\hline & $(90,67)$ & $(3,537)$ \\
\hline \multirow[t]{2}{*}{ La Voz } & $-367,3 * * *$ & $-11,00 * * *$ \\
\hline & $(90,92)$ & $(3,520)$ \\
\hline \multirow[t]{2}{*}{ Diario Uno } & $-370,5^{* * *}$ & $-12,28 * * *$ \\
\hline & $(91,11)$ & $(3,539)$ \\
\hline \multirow[t]{2}{*}{ Página/12 } & $-46,51$ & $15,75^{* * *}$ \\
\hline & $(135,0)$ & $(4,013)$ \\
\hline \multirow[t]{2}{*}{ Constant } & $409,8 * * *$ & $12,01 * * *$ \\
\hline & $(65,86)$ & $(2,577)$ \\
\hline Observations & 944 & 1092 \\
\hline R-squared & 0,080 & 0,085 \\
\hline
\end{tabular}

Errores estándar entre paréntesis

$* * * \mathrm{p}<0,01, * * \mathrm{p}<0,05, * \mathrm{p}<0,1$

unidad en la variable explicativa, es decir, que una noticia pase de ser sobre asuntos no públicos a ser sobre asuntos públicos, en promedio está asociado con un aumento de 174.2 interacciones en el nivel de engagement. Mientras que en el caso de Twitter, se asocia a un aumento de 12 interacciones en promedio en el nivel de engagement. Estos resultados muestran la existencia de una correlación entre la temática presentada en las noticias y el nivel de engagement por parte de los usuarios.

\section{Análisis cualitativo}

El análisis de las noticias que obtuvieron mayor engagement en Twitter (Cuadro 5), arroja que de las 10 mejor listadas, 8 pertenecen a la categoría asuntos públicos, in- 
Cuadro 5. Las 10 noticias más populares en Twitter durante el período analizado.

\begin{tabular}{|c|c|c|c|}
\hline Sitio & Título de la noticia & Temática & $\begin{array}{c}\text { Total } \\
\text { Engagement }\end{array}$ \\
\hline La Nación & $\begin{array}{l}\text { El Gobierno se niega a dialogar con la RAM y ratifica } \\
\text { que las fuerzas de seguridad estarán armadas durante } \\
\text { los operativos. }\end{array}$ & $\begin{array}{l}\text { Política } \\
\text { nacional }\end{array}$ & 708 \\
\hline Clarín & $\begin{array}{l}\text { Parado de manos y a } 25 \text { metros de altura, un experto } \\
\text { en clavados bate su propio récord pic.twitter.com/ } \\
\text { BowTCqCPjj }\end{array}$ & Sociedad & 500 \\
\hline La Nación & $\begin{array}{l}\text { La Iglesia no se salvó del recorte: el Gobierno le quitó } \\
\$ 137 \text { millones }\end{array}$ & $\begin{array}{l}\text { Política } \\
\text { nacional }\end{array}$ & 455 \\
\hline Perfil & $\begin{array}{l}\text { \# SUBMARINO | Los rostros y los nombres de los } 44 \\
\text { tripulantes del \#ARASanJuan . ¡Nuestros héroes! Los } \\
\text { estamos esperando pic.twitter.com/FRx12EGgJb }\end{array}$ & $\begin{array}{l}\text { Política } \\
\text { nacional }\end{array}$ & 407 \\
\hline$T N$ & $\begin{array}{l}\text { PASO 2017: Vidal y Carrió timbrearon sin Macri en } \\
\text { Mar del Plata }\end{array}$ & $\begin{array}{l}\text { Política } \\
\text { nacional }\end{array}$ & 400 \\
\hline$T N$ & $\begin{array}{l}\text { ¿Te gusta la nueva camiseta de Boca? Seguinos, usá } \\
\text { \# CamisetaBocaTN iy puede ser tuya! La sorteamos } \\
\text { mañana a las } 13 \text { en TN Deportivo. pic.twitter.com/ } \\
\text { uYQnGEP9qM }\end{array}$ & Deportes & 380 \\
\hline Perfil & $\begin{array}{l}\text { "Si bien no es ilegal juntarse a contar plata, todos } \\
\text { sabemos de que se trata". \# LaVerdad }\end{array}$ & $\begin{array}{l}\text { Política } \\
\text { nacional }\end{array}$ & 376 \\
\hline Página/12 & $\begin{array}{l}\text { ALERTA } \\
\text { La Corte Interamericana de DDHH ordenó reponer } \\
\text { la prisión domiciliaria de Milagro Sala pic.twitter. } \\
\text { com/6czoylzZZm }\end{array}$ & $\begin{array}{l}\text { Política } \\
\text { nacional }\end{array}$ & 319 \\
\hline$T N$ & $\begin{array}{l}\text { Cristina en Mar del Plata: "En esta Patria Grande } \\
\text { hay más diferencias entre un porteño y un jujeño, } \\
\text { que entre un jujeño y un peruano" pic.twitter.com/ } \\
\text { iEZAmvqxHN }\end{array}$ & $\begin{array}{l}\text { Política } \\
\text { nacional }\end{array}$ & 295 \\
\hline$T N$ & $\begin{array}{l}\text { Julio de Vido irá a indagatoria por la compra de } \\
\text { barcos de gas licuado }\end{array}$ & $\begin{array}{l}\text { Política } \\
\text { nacional }\end{array}$ & 256 \\
\hline
\end{tabular}

cluyendo noticias sobre decisiones del gobierno, los tripulantes de un submarino desaparecido, y casos de corrupción. Las dos restantes son sobre temas de asuntos no públicos -un experto en clavados y el sorteo de la camiseta del equipo de fútbol Boca Juniors. En el caso de Facebook, si bien en general las noticias con alto engagement son en su mayoría de asuntos públicos, y específicamente, sobre política, aparecen más noticias de asuntos no públicos con altos niveles engagement que en Twitter: 5 de las 10 noticias con más interacciones de usuarios pertenecen a esta categoría, incluyendo artículos sobre casos motivadores de historias personales que muestran a ciudadanos comunes en circunstancias extraordinarias - como el caso de una persona con un tipo sanguíneo con un anticuerpo inusual que sirve para generar una vacuna 
Cuadro 6. Las 10 noticias más populares en Facebook durante el período analizado.

\begin{tabular}{|c|c|c|c|}
\hline Sitio & Título de la noticia & Temática & $\begin{array}{c}\text { Total } \\
\text { Engagement }\end{array}$ \\
\hline Clarín & $\begin{array}{l}\text { Aníbal Fernández dice que no lo echaron del palco: } \\
\text { "Pasé a saludar y me fui"" }\end{array}$ & $\begin{array}{l}\text { Política } \\
\text { nacional }\end{array}$ & 12.958 \\
\hline La Nación & $\begin{array}{l}\text { Desde Educación Física hasta Filosofía, Luciano no } \\
\text { encuentra una carrera que le quite su hambre por el } \\
\text { conocimiento. }\end{array}$ & Sociedad & 8.706 \\
\hline Clarín & $\begin{array}{l}\text { El ex jefe de Gabinete } \mathrm{K} \text { intentó tener un lugar en el } \\
\text { escenario principal, pero lo bajaron. }\end{array}$ & $\begin{array}{l}\text { Política } \\
\text { nacional }\end{array}$ & 6.369 \\
\hline La Nación & $\begin{array}{l}\text { El exfuncionario kirchnerista, molesto, intentó } \\
\text { disuadir a los custodios del lugar, que con buenos } \\
\text { modos lo invitaron a retirarse hacia otro sector. }\end{array}$ & $\begin{array}{l}\text { Política } \\
\text { nacional }\end{array}$ & 4.206 \\
\hline Página/12 & $\begin{array}{l}\text { En una acción grotesca e inesperada, el Ministerio de } \\
\text { Salud bonaerense le pidió la renuncia "por cuestiones } \\
\text { políticas" a las directoras de la Maternidad Carlotto, } \\
\text { responsables de un modelo inclusivo y contracultural } \\
\text { único en Latinoamérica. }\end{array}$ & $\begin{array}{l}\text { Política } \\
\text { nacional }\end{array}$ & 4.017 \\
\hline La Nación & $\begin{array}{l}\text { ¡GROSO! Tiene } 22 \text { años y se consagró en el } \\
\text { Campeonato Mundial de la Pizza realizado en Parma, } \\
\text { Italia. }\end{array}$ & Sociedad & 3.676 \\
\hline Clarín & $\begin{array}{l}\text { El acto de los sindicatos terminó en escándalo. } \\
\text { Un grupo de militantes comenzó a insultar a los } \\
\text { dirigentes. }\end{array}$ & $\begin{array}{l}\text { Política } \\
\text { nacional }\end{array}$ & 3.630 \\
\hline La Nación & ¿Qué te parece el gesto del dueño del restaurante? & Sociedad & 3.097 \\
\hline La Nación & $\begin{array}{l}\text { Su sangre tiene un anticuerpo inusual que sirve para } \\
\text { generar una vacuna para los recién nacidos y es } \\
\text { donante en su pueblo hace más de } 60 \text { años. }\end{array}$ & $\begin{array}{l}\text { Ciencia y } \\
\text { Tecnología }\end{array}$ & 2.578 \\
\hline Diario Uno & $\begin{array}{l}\text { Sorteamos dos backstages exclusivos para estar } \\
\text { con en su show. Para participar dale me gusta } \\
\text { a nuestra fan page. Dejá tus } 3 \text { últimos números } \\
\text { del DNI Compartí este posteo El sorteo se realizará } \\
\text { el 13/11 }\end{array}$ & Sociedad & 2.319 \\
\hline
\end{tabular}

para los recién nacidos y es donante en su pueblo hace más de 60 años- o buscan la participación de los lectores a través de sorteos.

Lo que surge de la descripción de las noticias más populares en ambas redes sociales es que si bien los sitios ofrecen en su gran mayoría noticias sobre temáticas de asuntos no públicos, los usuarios parecen interactuar en mayor (o igual) medida con contenidos pertenecientes a asuntos públicos. Sin embargo, tanto Twitter como -y en mayor medida- Facebook, muestran noticias con historias de deportes, personales y de motivación, que también captan fuertemente la atención del público. 


\section{Conclusiones}

En la muestra analizada para este estudio, las cuentas oficiales de estos ocho sitios periodísticos en redes sociales presentaron una mayor cantidad de noticias sobre temáticas de asuntos no públicos que de noticias tradicionalmente denominadas de asuntos públicos por su vinculación con la vida política. Esto significa que la agenda que los sitios proponen en sus redes sociales muestra un cambio respecto de la tradicional preferencia de los medios informativos por sobre los asuntos públicos a la hora de construir su agenda (Boczkowski y Mitchelstein, 2013; Prior, 2007; Tewksbury 2003).

Sin embargo, las noticias de asuntos públicos generan mayor engagement entre los usuarios, más allá de la agenda propuesta por los sitios. El análisis de qué temáticas son las que generan mayor engagement muestra que existe una mayor interacción con noticias sobre asuntos públicos; además, podemos observar una pequeña diferencia en el comportamiento de ambas redes: mientras que en la muestra de Twitter 8 de las 10 noticias con mayor interacción son sobre asuntos públicos, en la de Facebook hay una proporción mayor que en la red anterior de noticias sobre esparcimiento, más puntualmente noticias que presentan historias de ciudadanos comunes, combinada con otras noticias de temáticas populares como deportes.

Estos hallazgos tienen implicancias tanto para los estudios sobre construcción de la agenda en redes sociales como para el análisis del compromiso cívico en nuevos medios de comunicación.

El predominio de noticias de asuntos no públicos sugiere un nuevo comportamiento por parte de los sitios, quienes asumen nuevas estrategias de producción de contenido al ampliar su agenda temática en redes sociales, tradicionalmente asociada a la alta prevalencia de los asuntos públicos. Como los medios se encuentran en un proceso de adaptación, es posible pensar una tendencia en los sitios a "ablandar" su agenda en las redes respecto de lo que muestran en los portales oficiales. Estos hallazgos también reflejan los múltiples lugares que hoy asumen las redes sociales en los consumos informativos y en las interacciones que los usuarios expresan en sus redes con los contenidos a los que se exponen.

El alto nivel de engagement con temas relacionados con asuntos públicos en comparación con los temas de asuntos no públicos se encuentra en línea con hallazgos que versan sobre un uso de redes para el compromiso cívico y la construcción de capital social, es decir, para la relación con contactos a partir de temas políticos y sociales y no solo individuales (Gil de Zúñiga et al., 2012; Lee y Ma, 2012). Los hallazgos de este estudio brindan cierto apoyo a esta distinción, en la medida que presentan una mayor propensión de los usuarios a interactuar con contenidos de índole pública, posiblemente provechosa para su imagen social y su imagen frente a otros.

La exposición a noticias sobre asuntos públicos no implica necesariamente una búsqueda activa, sino también una dinámica incidental (Boczkowski, Mitchelstein y Matassi, 2018), y se encuentra, muchas veces, relacionada con la interacción con otros usuarios, en donde se destacan las noticias compartidas como una fuente de información. En este sentido, es posible pensar que recomendaciones o el acto de compartir información vía redes pueden tener influencia positiva en el nivel de participación política (Effing, Van Hillegersberg, y Huibers, 2011; Valenzuela, 2013). 
Este estudio se inserta en el debate sobre la integración de los medios tradicionales en las redes sociales, así como también sobre el lugar que ocupan las redes en la búsqueda y exposición ante las noticias. Cada vez más usuarios se exponen a información en redes, pues la misma circula a gran escala en un ecosistema de permanente competencia por la atención de audiencias diversificadas e interactivas. Los usuarios se encuentran empoderados en su capacidad de producción y consumo informativo, cuestionando el lugar tradicional de los medios en la construcción de agenda. Las redes permiten reunir las facetas privada y pública de los usuarios, y ofrecen nuevas formas de consumo que se escapan de los límites tradicionales (Livingstone, 2008; Madden et al., 2013; Marwick y boyd, 2014; Towner y Dulio, 2012; Wu et al., 2011). Las dimensiones de producción y consumo informativo cambian con el uso de las redes sociales, en la medida que se entrelazan con cuestiones intra e interpersonales, moldeadas por las posibilidades de cada plataforma.

Un escenario en el cual las empresas que producen contenido periodístico distribuyen principalmente noticias de asuntos no públicos en sus redes sociales, pero generan más engagement a través de sus posteos de asuntos públicos es consistente con la caracterización de los y las consumidores de noticias como ciudadanos y ciudadanas monitoriales (Schudson, 1998), que vigilan de manera amplia el ambiente informativo para identificar posibles temas de importancia y reaccionar frente a ellos, en lugar de estar dedicados de manera permanente y deliberada a la búsqueda de información sobre asuntos públicos.

Este es un estudio exploratorio con algunas limitaciones que deben ser tenidas en cuenta. En primer lugar, al no tener acceso a la totalidad de los posteos en redes sociales de los ocho sitios, los hallazgos no nos permiten distinguir diferentes estrategias de los medios y reacciones del público por horario de posteo o circunstancias excepcionales, como elecciones o competencias deportivas globales. En segundo lugar, aunque observamos algunas tendencias en la producción y en la interacción con los contenidos, vale la pena analizar de forma pormenorizada cada una de las formas de interacción y observar las actitudes subyacentes en las redes (comentar, compartir, solo dar "me gusta", etc.). En tercer lugar, como el alcance real de las publicaciones no es de dominio público, resulta imposible calcular la "tasa de engagement" que permita calibrar niveles de compromiso con las noticias sobre cantidad de veces que fueron vistas o exhibidas.

En suma, los medios informativos se encuentran ante el desafío de mantener su capacidad de formación de agenda y de llegada a sus audiencias. Con la difusión masiva de las redes sociales no solo buscan obtener una buena recepción de sus contenidos, sino también entender a sus lectores y responder activamente. La posibilidad que ofrecen plataformas como Facebook o Twitter de identificar e interactuar con las audiencias se vuelve un escenario sin precedentes para los medios informativos. En este camino de adaptación a las nuevas plataformas, los sitios experimentan cambios en la construcción de su agenda y se adaptan a las nuevas dinámicas de producción que imponen los tiempos de las redes sociales. A su vez, los usuarios reaccionan de distintas maneras a las agendas propuestas, y de forma más activa a las noticias sobre asuntos públicos, lo que habilita a analizar las redes como un espacio para la sociabilidad pero también de ejercicio de la ciudadanía y el compromiso cívico. Esperamos que este trabajo constituya un paso fructífero en esa dirección. 


\section{Referencias}

Ardèvol-Abreu, A., \& Gil de Zuniga, H. (2017). Effects of editorial media bias perception and media trust on the use of traditional, citizen, and social media news. Journalism \& Mass Communication Quarterly, 94(3), 703-724.

Asur, S., Huberman, B. A., Szabo, G., \& Wang, C. (2011). Trends in social media: persistence and decay. Proceedings of the Fifth International AAAI Conference on Weblogs and Social Media (435-437).

Bachmann, I., \& de Zuniga, H. G. (2013). News platform preference as a predictor of political and civic participation. Convergence, 19(4), 496-512.

Bandari, R., Asur, S., \& Huberman, B. A. (2012). The pulse of news in social media: Forecasting popularity. Proceedings of the Sixth International AAAI Conference on Weblogs and Social Media (435-437), 12, 26-33.

Boczkowski, P. J., \& Mitchelstein, E. (2012). How users take advantage of different forms of interactivity on online news sites: Clicking, e-mailing, and commenting. Human Communication Research, 38(1), 1-22.

Boczkowski, P. J., \& Mitchelstein, E. (2013). The news gap: When the information preferences of the media and the public diverge. Cambridge: MIT Press.

Boczkowski, P. J., Mitchelstein, E., \& Matassi, M. (2018). "News comes across when I'm in a moment of leisure": Understanding the practices of incidental news consumption on social media. New Media \& Society.

boyd, D., Golder, S., \& Lotan, G. (2010). Tweet, tweet, retweet: Conversational aspects of retweeting on twitter. Proceedings of the 43rd Hawaii international conference on System Sciences (pp. 1-10).

Conway, B. A., Kenski, K., \& Wang, D. (2015). The rise of Twitter in the political campaign: Searching for intermedia agenda-setting effects in the presidential primary. Journal of Computer-Mediated Communication, 20(4), 363-380.

Conway-Silva, B. A., Filer, C. R., Kenski, K., \& Tsetsi, E. (2017). Reassessing Twitter's agenda-building power: An analysis of intermedia agenda-setting effects during the 2016 presidential primary season. Social Science Computer Review.

Effing, R., Van Hillegersberg, J., \& Huibers, T. (2011). Social media and political participation: are Facebook, Twitter and YouTube democratizing our political systems? In International conference on electronic participation (pp. 25-35). Berlin: Springer.

Fletcher, R., \& Nielsen, R. K. (2018). Are people incidentally exposed to news on social media? A comparative analysis. New Media \& Society, 20(7), 2450-2468.

Gil de Zúñiga, H., Jung, N., \& Valenzuela, S. (2012). Social media use for news and individuals' social capital, civic engagement and political participation. Journal of ComputerMediated Communication, 17(3), 319-336.

Gil de Zúñiga, H., Molyneux, L., \& Zheng, P. (2014). Social media, political expression, and political participation: Panel analysis of lagged and concurrent relationships. Journal of Communication, 64(4), 612-634.

Gottfried, J., \& Shearer, E. (2016). News Use Across Social Medial Platforms 2016. Pew Research Center.

Hermida, A., Fletcher, F., Korell, D., \& Logan, D. (2012). Share, like, recommend: Decoding the social media news consumer. Journalism Studies, 13(5-6), 815-824.

Hester, J. B., \& Dougall, E. (2007). The efficiency of constructed week sampling for content analysis of online news. Journalism \& Mass Communication Quarterly, 84(4), 811-824. 
Hollebeek, L. D., Glynn, M. S., \& Brodie, R. J. (2014). Consumer brand engagement in social media: Conceptualization, scale development and validation. Journal of interactive marketing, 28(2), 149-165.

Holton, A. E., Baek, K., Coddington, M., \& Yaschur, C. (2014). Seeking and sharing: Motivations for linking on Twitter. Communication Research Reports, 31(1), 33-40.

Krippendorff, K., \& Bock, M. A. (2009). The content analysis reader. Thousand Oaks: Sage.

Lee, J. G., Moon, S., \& Salamatian, K. (2010). An approach to model and predict the popularity of online contents with explanatory factors. In Web Intelligence and Intelligent Agent Technology (WI-IAT), 2010 IEEE/WIC/ACM International Conference on (Vol. 1, pp. 623-630).

Lee, C. S., \& Ma, L. (2012). News sharing in social media: The effect of gratifications and prior experience. Computers in human behavior, 28(2), 331-339.

Lim, M. (2012). Clicks, cabs, and coffee houses: Social media and oppositional movements in Egypt, 2004-2011. Journal of communication, 62(2), 231-248.

Livingstone, S. (2008). Taking risky opportunities in youthful content creation: teenagers' use of social networking sites for intimacy, privacy and self-expression. New Media \& Society, 10(3), 393-411.

Ma, L., Lee, C. S., \& Goh, D. H. L. (2011). That's news to me: the influence of perceived gratifications and personal experience on news sharing in social media. In Proceedings of the 11th annual international ACM/IEEE joint conference on Digital libraries (pp. 141144).

Madden, M., Lenhart, A., Cortesi, S., Gasser, U., Duggan, M., Smith, A., \& Beaton, M. (2013). Teens, social media, and privacy. Pew Research Center, 21, 2-86.

Martínez, F. (2010). La teoría de los usos y gratificaciones aplicada a las redes sociales. Libro de actas del II Congreso Internacional Comunicación. Salamanca: Universidad de Salamanca.

Marwick, A. E., \& boyd, D. (2014). Networked privacy: How teenagers negotiate context in social media. New Media \& Society, 16(7), 1051-1067.

Meraz, S. (2009). Is there an elite hold? Traditional media to social media agenda setting influence in blog networks. Journal of Computer-Mediated Communication, 14(3), 682707.

Messing, S., \& Westwood, S. J. (2014). Selective exposure in the age of social media: Endorsements trump partisan source affiliation when selecting news online. Communication Research, 41 (8), 1042-1063.

Mitchelstein, E., \& Boczkowski, P. J. (2018). Juventud, estatus y conexiones. Explicación del consumo incidental de noticias en redes sociales. Revista mexicana de opinión pública, (24), 131-145.

Newman, N., Fletcher, R., Kalogeropoulos, A., Levy, D., \& Kleis Nielsen, R. (2018). Reuters digital news report 2018. Reuters Institute for the Study of Journalism, University of Oxford.

Nielsen, R. K., \& Schrøder, K. C. (2014). The relative importance of social media for accessing, finding, and engaging with news: An eight-country cross-media comparison. Digital journalism, 2(4), 472-489.

Oeldorf-Hirsch, A., \& Sundar, S. S. (2015). Posting, commenting, and tagging: Effects of sharing news stories on Facebook. Computers in Human Behavior, 44, 240-249.

Oh, S., \& Syn, S. Y. (2015). Motivations for sharing information and social support in social media: A comparative analysis of Facebook, Twitter, Delicious, YouTube, and Flickr. Journal of the Association for Information Science and Technology, 66(10), 2045-2060. 
Powers, M., \& Vera-Zambrano, S. (2017). How journalists use social media in France and the United States: Analyzing technology use across journalistic fields. New Media \& Society, 1461444817731566.

Prior, M. (2007). Post-broadcast democracy: How media choice increases inequality in political involvement and polarizes elections. Cambridge University Press.

Quan-Haase, A., \& Young, A. L. (2010). Uses and gratifications of social media: A comparison of Facebook and instant messaging. Bulletin of Science, Technology \& Society, 30(5), 350-361.

Rainie, L., Smith, A., Schlozman, K. L., Brady, H., \& Verba, S. (2012). Social media and political engagement. Pew Internet \& American Life Project.

Raimondo Anselmino, N., Sambrana, A. M., \& Cardoso, A. L. (2017). Medios tradicionales y redes sociales en Internet: un análisis de los posteos compartidos por los diarios argentinos Clarín y La Nación en Facebook (2010-2015). Astrolabio, (19), 32-68.

Riffe, D., Aust, C. F., \& Lacy, S. R. (1993). The effectiveness of random, consecutive day and constructed week sampling in newspaper content analysis. Journalism Quarterly, 70(1), 133-139.

Sakaki, T., Okazaki, M., \& Matsuo, Y. (2010). Earthquake shakes Twitter users: real-time event detection by social sensors. In Proceedings of the 19th international conference on World Wide Web (pp. 851-860). Raleigh, North Carolina: ACM.

Schudson, M. (1998). The good citizen. Cambridge: Harvard University Press.

Stassen, W. (2010). Your news in 140 characters: Exploring the role of social media in journalism. Global Media Journal-African Edition, 4(1), 116-131.

Sunstein, C. R. (2018). \# Republic: Divided democracy in the age of social media. Princeton: Princeton University Press.

Tewksbury, D. (2003). What do Americans really want to know? Tracking the behavior of news readers on the Internet. Journal of communication, 53(4), 694-710.

Towner, T. L., \& Dulio, D. A. (2012). New media and political marketing in the United States: 2012 and beyond. Journal of Political Marketing, 11(1-2), 95-119.

Turcotte, J., York, C., Irving, J., Scholl, R. M., \& Pingree, R. J. (2015). News recommendations from social media opinion leaders: Effects on media trust and information seeking. Journal of Computer-Mediated Communication, 20(5), 520-535.

Valenzuela, S. (2013). Unpacking the use of social media for protest behavior: The roles of information, opinion expression, and activism. American Behavioral Scientist, 57(7), 920-942.

Valeriani, A., \& Vaccari, C. (2017). Political talk on mobile instant messaging services: a comparative analysis of Germany, Italy, and the UK. Information, Communication \& Society, 1-17.

Vargo, C. J., Guo, L., McCombs, M., \& Shaw, D. L. (2014). Network issue agendas on Twitter during the 2012 US presidential election. Journal of Communication, 64(2), 296-316.

Wanta, W., Golan, G., \& Lee, C. (2004). Agenda setting and international news: Media influence on public perceptions of foreign nations. Journalism \& Mass Communication Quarterly, 81(2), 364-377.

Wasike, B. S. (2013). Framing news in 140 characters: How social media editors frame the news and interact with audiences via Twitter. Global Media Journal, 6(1), 5.

Weeks, B. E., \& Holbert, R. L. (2013). Predicting dissemination of news content in social media: A focus on reception, friending, and partisanship. Journalism \& Mass Communication Quarterly, 90(2), 212-232. 
Whiting, A., \& Williams, D. (2013). Why people use social media: a uses and gratifications approach. Qualitative Market Research: An International Journal, 16(4), 362-369.

Wu, S., Hofman, J. M., Mason, W. A., \& Watts, D. J. (2011). Who says what to whom on twitter. In Proceedings of the 20th international conference on World Wide Web (pp. 705714). 
